\title{
Mycobacterium triviale Presenting as Endobronchial Lesion in a Young Immunocompetent Girl
}

\section{Nabil Ghaleb}

Department of Medicine, College of Medicine, King Khaled University Hospital, KSU, Riyadh, Saudi Arabia

\begin{abstract}
The Mycobacterium terrae complex, consisting of three saprophytic species, (M. terrae, M. nonchromogenicum, and $M$. triviale), they rarely cause diseases in immunocompetent people. There are few case reports of lung involvement by Mycobacterium terrae complex. We reported a 13 years old immunompetent girl with a cavitary opacity seen on chest imaging and bronchoscopy revealed an endobronchial lesion, sputum and tissue specimens revealed growth of Mycobacterium triviale. Our patient is unique as it is the first case reported in Saudi Arabia with this type of Mycobacteria, in addition there is no case reported in the literature with Mycobacterium triviale with endobronchial lesion, the patient treated with a combination of rifampicin, ciprofloxacin and ethambutol for six months, she showed good response with resolution of radiological abnormalities and she did not have relapse after one year of follow up.
\end{abstract}

Keywords: Immunompetent girl; Mycobacterium triviale; Bronchial asthma

\section{Case Report}

A 13 years old girl referred to king Khalid university hospital, Riyadh, from another hospital, she is a known case of bronchial asthma on PRN inhalers, the patient had history of recurrent chest infections since 7 years, in the referring hospital the patient was admitted with 3 weeks history of productive cough, dyspnea, fever and chest discomfort. There was no history of hemoptysis, anorexia, weight loss or night sweats, no history of contact with sick person or exposure to pets or animals, she has no previous Hx of extra pulmonary infections. In the referring hospital she was managed as community acquired pneumonia with cefuroxim and moxifloxacin, the patient showed improvement of symptoms. In the referring hospital the sputum for AFB was positive but microscopy showed long branching filaments suspicious of Nocardia and sputum PCR for Mycobacterium tuberculosis was negative and because of residual abnormality on chest $\mathrm{X}$-ray and CT lungs which raised possibility of underlying lymphoma, the patient referred to our hospital for further intervention, the patient had history of Bell's palsy, she left the school because of poor performance, she is living with her family in Riyadh with an average social class. Examination in our hospital revealed young girl, thin and short for her age, pale but looking will, fully conscious, not dyspnic, she is a febrile and other vital signs were normal, there was no clubbing or preferal lymphadenopathy, chest exam revealed decrease breath sounds on the right side otherwise normal, other systemic examination was normal. Initial investigations revealed CBC: WBC $7.1 \mathrm{Hb} 10$ platelets normal ESR : 95, urea and electrolytes and LFT are normal, chest X-ray (Figure 1) showed right paratracheal and hilar lymphadenopathy with right parahilar cavitary airspace pneumonic opacity, CT lungs (Figure 2) showed right lower lobe consolidation with cavitation with bilateral hilar lymphadenopathy.

In our hospital, sputum for AFB was also positive and culture was positive for atypical Mycobacteria after one week bronchoscopy showed Shiny mass bleed to touch blocking most of the Rt main bronchous, $\mathrm{BAL}$ was done but $\mathrm{Bx}$ was not taken because of the risk of bleeding, BAL results revealed No AFB But culture was also +ve for atypical Mycobacteria, Cytology revealed several polymorphs consistent with inflammatory process because an underlying lymphoma couldn't be ruled out the pt. underwent Rt side thoracotomy, which revealed multiple mediastinal caseating lymph nodes which were eroding to the lumen of the Rt. Main and Rt. Upper lobe bronchi which were explored and lymph nodes specimens taken for investigation. Histopathology results of the specimens revealed: Reactive follicular lymphoid hyperplasia with histiocytic proliferation with mixed inflammation, no malignant cells, there are filamentous acid fast bacilli organisms suggestive of either nocardiosis or atypical mycobacteria (Figure 3), From TB lab Bx stain revealed :long filamentous AFB, Culture was positive for atypical mycobacteria which is sensitive to Rifampicin ,ethambutol but resistant to isonized and Streptomycin and the specimens were send to another hospital for further processing. Pt was referred to infectous group who started the patient on Rifampicin, Clarithromycin, Ciprofloxacin and etahmbutol. Pt was discharged after 2 weeks in good condition.

After about one month, report of the molecular and biochemical processing of the specimen revealed Mycobacterial triviale. Patient was seen monthly in the thoracic surgery and ID clinic, clinically she was doing well and follow up chest $\mathrm{X}$ ray showed progressive resolution of the abnormal findings. In the first visit in the clinic, clarithromycin was discontinued and the patient was continued on rifampicin, ciprofloxacin and ethambutol for six months, she gained weight and ESR came back to normal, after completion of treatment she was followed for one year. She did not show any evidence of relapse either clinically or radiologicaly (Figure 4).

\section{Discussion}

The original description of the Mycobacterium terrae complex subspecies was by Richmond and cummings in 1955 [1]. The Mycobacterium terrae complex includes M. terrae, Mycobacterium nonchromogenicum, and Mycobacterium triviale [2]. Members of the $M$. terrae complex are Runyon group III, non-pigmented, slowgrowing organisms. Because of the presumed nonpathogenic nature of these organisms, there has been little effort to distinguish the species of this complex in the clinical setting [3]. M. terrae organisms are much

Corresponding author: Dr. Nabil Ghaleb, MBBS, FCCP, Pulmonary Division, Dept of Medicine, College of Medicine, King Khaled University, Hospital, KSU 11472, P.O. Box 7805, E-mail : kkuh2005@yahoo.com

Received September 08, 2011; Accepted November 29, 2011; Published December 02, 2011

Citation: Ghaleb N (2011) Mycobacterium triviale Presenting as Endobronchia Lesion in a Young Immunocompetent Girl. J Pulmonar Respirat Med 1:109. doi:10.4172/2161-105X.1000109

Copyright: ( ) 2011 Ghaleb N. This is an open-access article distributed under the terms of the Creative Commons Attribution License, which permits unrestricted use, distribution, and reproduction in any medium, provided the original author and source are credited. 
less common mycobacterial pathogens than are other nontuberculous mycobacteria at any site of infection [4]. Up to $18 \%$ of isolates of nontuberculous mycobacteria that are recovered from humans are $M$. terrae complex organisms [5,6] and these isolates are rarely causing clinical disease, thus signifying nonpathogenic colonization [7]. But despite the common opinion that M. terrae complex isolates are nonpathogenic, these organisms are occasionally identified in the clinical laboratory in the setting of clinical disease of the joints, tendons (tenosynovitis), lungs, gastrointestinal tract, and genito-urinary tract.

There are few case reports of human disease that involve the lungs caused by $M$. terrae complex organisms [8-15], in a review by scott smith and colleagues of a total of 54 cases with $M$. terrae complex diseases, Lung disease was found in $26 \%$ of cases ( 14 patients out of 54 ), in many of the reports of the case series of this review members of $M$. terrae complex were not identified. In this review no predisposing factors are found in $44 \%$ of patients with disease caused by $M$ terrae complex and there was no correlation between the presence of a comorbid condition and an undesirable outcome in these cases [4]. Pulmonary infection with $M$ terrae can result in a cavitary process with noncaseating granulomas in tissue samples [16]. Following this review there is only one further case of Mycobacterium terrae involving the lung reported in spain by Diaz ricoma [17] and another case reported by carbonara with disseminated $M$. terrae infection involving the lung in advanced HIV patient [7]. in the literature there is no case reported with lung disease by $M$. terrae complex; identified the member Mycobacterium triviale, though it may be part of the cases reported with $M$. terrae complex disease, in addition there is no case reported with $M$. terrae complex involving the lung with endobronchial lesion.

The optimum antimicrobial therapy for $M$. terrae complex has

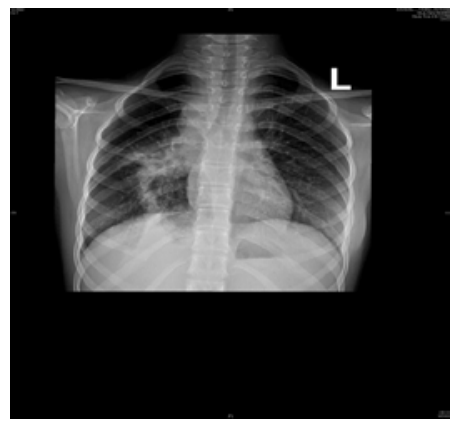

Figure 1: Chest roentgenogram before surgical intervention showing right paratracheal and hilar lymphadenopathy with right parahilar cavitary airspace pneumonic opacity.
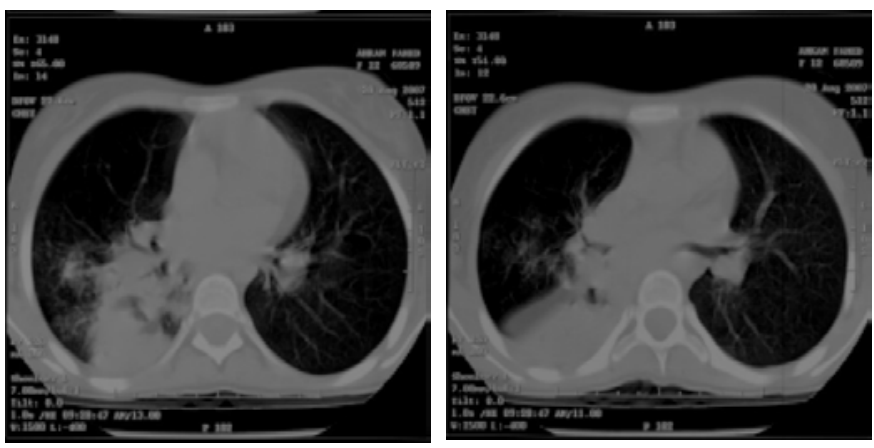

Figure 2: Two CT scan sections at lower zones showing right lower lobe consolidation with cavitation with bilateral hilar lymphadenopathy.

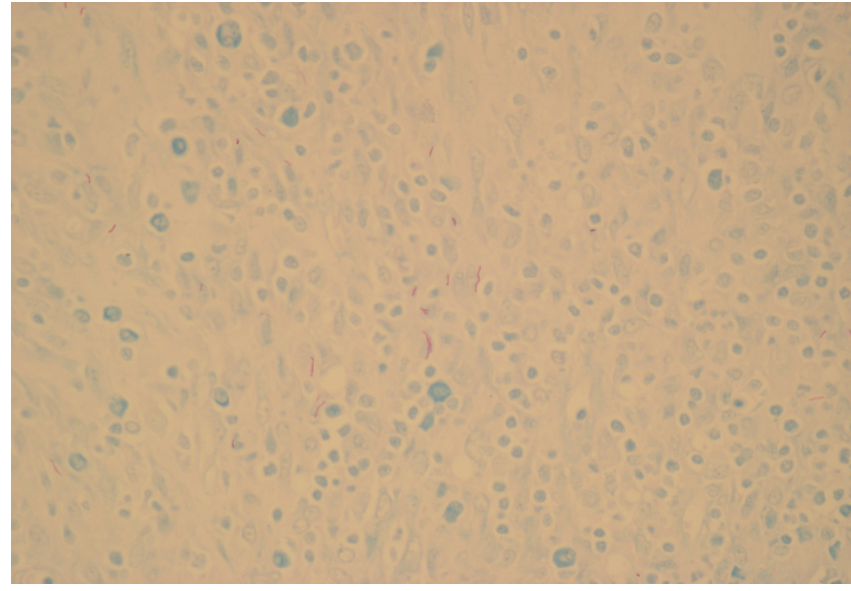

Figure 3: Mediastinal lymph node biopsy showing long filamentous acid fast organisms.

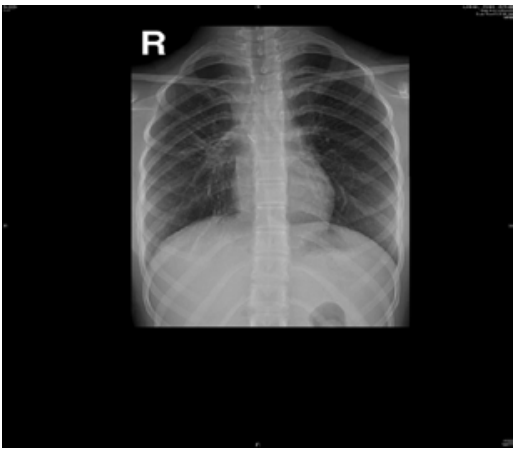

Figure 4: Chest roentgenogram one year after completion of treatment showing almost resolving of the previous abnormalities.

not been established [18]. In vitro antibiotic susceptibility testing is preferred for better selection of the best regimen of therapy, which may have to be continued for a prolonged period to increase the possibility of cure. Treatment with clarithromycin, ethambutol, and rifampicin was recommended and the duration of treatment needs to be for at least 12 months after clinical response [4].

Our patient had recurrent chest infections, which were probably due to post obstructive pneumonia due to obstruction of the right main bronchus by the endobronchial lesion, the patient acute symptoms responded initially to the pneumonia treatment; but her radiological abnormalities, her history of recurrent pneumonia and the presence of endobronchial lesion raised the possibility of chronic underling disease and though the patient sputum grown atypical Mycobacteria which can alone explain the radiological findings, the possibility of underling lymphoma still couldn't be ruled out especially it could be a risk factor for this unusual infection there is reported cases of lymphoma with endobronchial lesion; we thought of this rare possibility in this patient and the growth of these organisms could be just a contaminant; but the possibility of a real infection with atypical Mycobacteria with underlying lymphoma could not be completely excluded.

Giving the patient medical treatment for atypical Mycobacteria and observing for the response was an option but because the treatment usually will takes long time it would not be a favorable option if the patient had an underlying lymphoma, so we preferred to perform right sided thoracotomy and exploration to take good biopsies mainly to rule out lymphoma in addition to relieve the bronchial obstruction. 
Citation: Ghaleb N (2011) Mycobacterium triviale Presenting as Endobronchial Lesion in a Young Immunocompetent Girl. J Pulmonar Respirat Med 1:109. doi:10.4172/2161-105X.1000109

Page 3 of 3

In conclusion we add to the literature another case with lung disease involved by the member of $M$. terrae complex; Mycobacterium trivialae and it is the first reported case with endobronchial lesion with this organism.

\section{References}

1. Richmond L, Cumming MM (1950) An evaluation of methods of testing acidfast bacilli. Am Rev of Tuberc 62: 632-637.

2. Tortoli $E$ (2003) Impact of genotypic studies on mycobacterial taxonomy: the new mycobacteria of the 1990's. Clin Microbiol Rev 2: 319-354.

3. Torkko P (1998) Separation among species of Mycobacterium terrae complex by lipid analyses: comparison with biochemical tests and $16 \mathrm{~S}$ rRNA sequencing. J Clin Microbiol 36: 499-505

4. Smith DS, Lindholm-Levy P, Huitt GA, Heifets LB, Cook JL (2000) Mycobacterium terrae: case reports, literature review, and in vitro antibiotic susceptibility testing. Clin Infect Dis 30: 444-453.

5. Shafer RW, Sierra MF (1992) Mycobacterium xenopi, Mycobacterium fortuitum, Mycobacterium kansasii, and other nontuberculous mycobacteria in an area of endemicity for AIDS. Clin Infect Dis 15: 161-162.

6. Bahrmand AR, Madani H, Samar G, Khalilzadeh L, Bakayev VV, et al. (1996) Detection and identification of non-tuberculous mycobacterial infections in 6472 tuberculosis suspected patients. Scand J Infect Dis 28: 275-278.

7. Carbonara S (2000) Disseminated Mycobacterium terrae infection in a patient with advanced human immunodeficiency virus disease. Clin Infect Dis 30: 831835

8. Tsukamura M, Kita N, Otsuka W, Shimoide H (1983) A study of the taxonomy of the Mycobacterium nonchromogenicum complex and report of six cases of lung infection due to Mycobacterium nonchromogenicum. Microbiol Immunol 27: 219-236.

9. Kuze F, Mitsuoka A, Chiba W, Shimizu Y, Ito M, et al. (1983) Chronic pulmonary infection caused by Mycobacterium terrae complex: a resected case. Am Rev Respir Dis 128: 561-565.

10. Krisher KK, Kallay MC, Nolte FS (1988) Primary pulmonary infection caused by Mycobacterium terrae complex. Diagn Microbiol Infect Dis 11: 171-175.

11. Palmero DJ, Teres RI, Eiguchi K (1989) Pulmonary disease due to Mycobacterium terrae. Tubercle 70: 301-303.

12. Tonner JA, Hammond MD (1989) Pulmonary disease caused by Mycobacterium terrae complex. South Med J 82: 1279-1282.

13. Spence TH, Ferris VM (1996) Spontaneous resolution of a lung mass due to infection with Mycobacterium terrae. South Med J 89: 414-416.

14. Peters EJ, Morice R (1991) Miliary pulmonary infection caused by Mycobacterium terrae in an autologous bone marrow transplant patient. Chest 100: $1449-1450$.

15. Mayo J, Collazos J, Martinez E (1998) Mycobacterium nonchromogenicum bacteremia in an AIDS patient. Emerg Infect Dis 4: 124-125.

16. García García JM, Palacios Gutiérrez JJ, Sánchez Antuña AA (2005) Respiratory Infections Caused By Environmental Mycobacteria. Arch Bronconeumol 41: 206-219.

17. Díaz Ricomá N, González Vargas F, Casado Moreno I, Galán Antoñanza L, Rojas Sierra M, et al. (2001) Infección pulmonar por Mycobacterium terrae. Arch Bronconeumol 37: 96-98.

18. Griffith DE, Aksamit T, Brown-Elliott BA, Catanzaro A, Daley C, et al. (2007) An Official ATS/IDSA Statement: Diagnosis, Treatment and Prevention of Nontuberculous Mycobacterial Diseases. Am J Respir Crit Care Med 175: 367 416. 